\title{
Itinéraires Itinéraires
}

Littérature, textes, cultures

2017-3 | 2018

Littératures expérimentales

\section{« Non-littérature »?}

"Non-literature"?

Gaëlle Théval

\section{OpenEdition}

Journals

Édition électronique

URL : https://journals.openedition.org/itineraires/3900

DOI : $10.4000 /$ itineraires.3900

ISSN : 2427-920X

Éditeur

Pléiade

\section{Référence électronique}

Gaëlle Théval, « « Non-littérature »? », Itinéraires [En ligne], 2017-3 | 2018, mis en ligne le 15 juin 2018, consulté le 21 septembre 2021. URL : http://journals.openedition.org/itineraires/3900 ; DOI : https:// doi.org/10.4000/itineraires.3900

Ce document a été généré automatiquement le 21 septembre 2021.

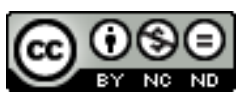

Itinéraires est mis à disposition selon les termes de la licence Creative Commons Attribution - Pas d'Utilisation Commerciale - Pas de Modification 4.0 International. 


\title{
« Non-littérature »?
}

\author{
"Non-literature"?
}

\section{Gaëlle Théval}

C'est ainsi, peut-être, qu'à l'image de ce qui a été qualifié de NON-ART ces dernières années, au regard de la peinture conventionnelle, mais qui n'en a pas moins constitué sa part la plus active, la plus vivante et bouleversante, et paradoxalement ainsi, sa continuité même, la poésie sonore peut apparaître, de par ses « pratiques » et « supports », lois et disciplines, comme de la NON-

LITTÉRATURE.

Beaucoup plus qu'une simple sortie du livre, la poésie opérait donc une sortie bien plus radicale, celle de la littérature, de sa vieillerie et de ses scléroses, de son retard au regard des arts plastiques ayant poursuivi dans la voie de la modernité et de l'expérimentation.

Un demi-siècle sépare les pratiques décrites par ces constats. Le premier, formulé par Heidsieck (2001a : 242), décrit le mouvement amorcé au cours des années 1950 par la poésie sonore, mais aussi les poésies visuelles, et de performance, parfois regroupées sous le nom «poésies expérimentales ». Le second, de Ruffel (2010: 61), désigne des pratiques apparues au mitan des années 1990, qui ne s'affublent pas d'épithètes mais sont aussi parfois ainsi nommées. En 2010, Ruffel (65) peut observer une "généralisation de ces pratiques au-delà des seuls territoires de la "littérature d'avant-garde", [...] leur multiplicité et leur caractère massif », raison d'être du numéro que la revue Littérature consacre aux «littératures exposées», «ces pratiques littéraires multiples (performances, lectures publiques, interventions sur le territoire, travaux sonores et visuels) pour lesquelles le livre n'est plus un but ni un prérequis » (Rosenthal et Ruffel $2010: 5)$. 
2 Il s'est donc, semble-t-il, produit quelque chose, d'assez massif pour que les études littéraires finissent par s'ouvrir à des pratiques longtemps exclues de leur champ. Comme si la sortie de la poésie hors de la " littérature » avait entraîné avec elle la littérature ellemême, de la même manière que ce qu'on a pu appeler le "non-art ${ }^{1}$ ", dans une lignée post-duchampienne, a fini par entraîner l'art hors de ses limites assignées, l'obligeant à se redéfinir selon de nouveaux paradigmes, d'une manière qui peut cependant paraître décalée. Non que le mouvement ne se soit, pourtant, amorcé au même moment dans les pratiques, soit au cours des années 1950. Mais l'état du champ en aura semble-t-il freiné la réception, occulté les enjeux. Ainsi Castellin (2001: 118), retraçant l'histoire de la revue DOC(K)S, peut écrire qu'en 1976 encore, le textualisme de Tel Quel tient «le haut du pavé littéraire "avant-gardiste" ». Ce n'est que dans les années qui suivent "que s'esquissera un changement pour déboucher vingt ans plus tard seulement sur une reconnaissance générale ». Une réception « à retard », donc, à l'instar de celle qui fut réservée au readymade duchampien, dont les conséquences pleines ne se sont révélées que des décennies après son irruption. Cela crée parfois quelques illusions d'optique, amenant à prêter aux poésies des années 1990 des gestes de rupture déjà effectués dans les années $1960^{2}$. C'est bien davantage d'une reconnaissance, et d'une continuation qu'il s'agit. La réédition par Al Dante de l'œuvre de Heidsieck en est un signe, pensée par Cauwet comme une manière de rappeler "d'où viennent ces poésies ${ }^{3}$ ", mais aussi la présence active de ces poètes « historiques » (Heidsieck, Chopin, Bory, Blaine) dans le champ, en lien et dialogue avec la génération alors émergente. Réfléchir sur les pratiques contemporaines mérite une mise en perspective qui permette de mesurer l'apport des poésies expérimentales historiques au sein de ce mouvement généralisé.

De la "non-littérature » à la "littérature exposée » se serait produit quelque chose comme une " dé-définition ». La teneur du débat suscité par le développement de ce type d'œuvres n'est pas sans rappeler celle des querelles dont l'art contemporain a été le théâtre depuis les années 1990, dont les fondements critiques sont concomitants à la naissance des pratiques « non-art » (Vallier 1969). Il n'est que de lire l'attaque de Roubaud en 2010, pour être frappé par le retour d'arguments comparables, conduisant à dénier aux pratiques décrites le statut de poésie : « Pourquoi les baptiser "poésie" ? Pourquoi ne pas les nommer musique, gymnastique, air d'opéra, numéro de cirque, sketch, chanson, ballet, strip-tease ?» Du côté des praticiens / théoriciens des formes attaquées le constat n'est pas si différent quant à leur caractère non «littéraire ». Imaginant un lecteur découvrant $D O C(K) S$, Castellin $(2001: 22)$ insiste sur le déroutement qu'il ne peut manquer de ressentir: "“ça, de la poésie ?!!!!” ». Devant des objets plus immédiatement contemporains, Hanna (2010:7) revient sur la métaphore des Objets verbaux non identifiés fréquemment utilisée depuis sa mise en circulation par Cadiot et Alféri en 1995, pour préciser :

Ces objets non identifiables, en effet, sont à comprendre comme les représentants d'écritures dont le fonctionnement propre nécessite, plus encore que leur «dédéfinition » formelle en tant qu'écrits littéraires, leur désinstitutionnalisation, autrement dit leur sortie, au moins momentanée, du champ littéraire.

4 Le paradigme dominant ne permet pas d'identifier ces objets comme "littéraires", entrainant une forme de "stupeur critique " (Hanna 2010: 10). Le constat résonne avec celui de Cauquelin (1996: 13) sur les œuvres d'art contemporain : "Il semble que les critères par lesquels nous pouvions, il n'y guère encore, reconnaître des objets comme œuvres d'art ne soient plus appropriés à ce qui est là, devant nous, maintenant. » Et la 
philosophe de préciser qu'il ne s'agit pas d'opinions mais « de tout l'appareil, du dispositif global de nos croyances en quelque chose comme de l'art », formant une « doxa ».

Partant de la réception critique de ces pratiques, représentative d'une " doxa », il s'agirait alors de tenter de mesurer ce qui, dans les définitions, est amené à se déplacer : où le terme de «littérature " est, comme celui d'art, tenu pour un concept tantôt évaluatif, tantôt essentialiste, tantôt institutionnaliste, là où celui de " poésie » est plus volage. Les poésies expérimentales des années 1960 s'attribuent les épithètes " visuelle ", « sonore ", " élémentaire », "action », prolifération qui tient de la volonté de se distinguer dans un champ donné, mais aussi de s'y maintenir, alors même que leur identification ne va plus de soi. Castellin leur préfère le nom poésies, pointant leur relation problématique avec l'histoire de la poésie, et leur appartenance à cette dernière. La revendication du « terrible label » (Heidsieck 2004), fait qualifier ces pratiques de « néo-poésies » par Gleize (2009: 59), pour qui la négation implique in fine une "suraffirmation de la poésie» comme recherche permanente. Elle ne semble en revanche plus être un enjeu majeur pour une certaine frange de la création contemporaine qui se voit alors nommée "postpoésie ». Le nom de "poésie» est alors attribué comme par défaut, mais l'adjectif "poétique " perdure: Hanna parle de « dispositifs poétiques », Lebovici de "documents poétiques", au prix d'un transfert qui désambiguïse leur rapport au littéraire et sanctionne leur « désinstitutionnalisation ».

Pour envisager le lien des pratiques expérimentales et contemporaines à ces notions, le propos se concentrera sur deux figures, Bernard Heidsieck et Anne-James Chaton. Ce dernier, actif depuis la fin des années 1990 est considéré comme "héritier» du premier - et cultive la relation, évoquant l'importance de l'écoute de Vaduz ${ }^{4}$ dans son entrée en poésie, publiant « À l'Est de Vaduz », dans TIJA en 2000, postfaçant le recueil des Tapuscrits de Heidsieck, et se revendiquant "poète sonore", là où d'autres de sa génération, tout en utilisant des moyens communs à la poésie sonore, ne se nomment pas comme tel. Cependant, Chaton est aussi parfois désigné comme un représentant de la "post-poésie», et parmi ses nombreuses activités, seules certaines étaient jusqu’à récemment étiquetées " poésie sonore ${ }^{5}$ » sur l'ancien site de l'auteur, d'autres ayant trait aux collaborations avec des musiciens, à des activités plastiques ou à la scène. En cela, ces deux poètes, de deux générations différentes, sont assez représentatifs d'une évolution quant à la question générique vers une forme d'indifférence. Quels sont, au regard de ces deux œuvres, les traits paradigmatiques du « littéraire » qui semblent chuter?

\section{Poésies hors du livre?}

7 Pour Heidsieck la poésie sonore est de la «non-littérature » d'abord par sa sortie du médium traditionnellement associé au littéraire. Rosenthal et Ruffel (2010: 5) remarquent aussi dans l'introduction de leur ouvrage que «l'exposition déjoue le mode de reconnaissance de la littérature par l'imprimé et par le livre ».

8 Les raisons en seraient d'abord liées à un état moribond du champ et au manque de diffusion de la poésie, menant à la multiplication des lectures publiques, et à l'investissement de lieux institutionnels nouveaux. Ce constat est déjà celui que fait Heidsieck en 1955, date de sortie de son recueil Sitôt dit, chez Seghers. La publication chez un éditeur de poésie reconnu est concomitante de la prise de conscience du fonctionnement d'un milieu dans lequel les plaquettes s'échangent entre poètes, où les 
«lectures» se font en cercle fermé. Le livre fétichisé est fustigé comme espace d'une poésie réflexive devenue narcissique, emportée par un mouvement «centrifuge » qui la menace d'«asphyxie blanche» (Heidsieck 2001a: 131) et véhicule des connotations désuètes, poussiéreuses, signes d'un déphasage d'avec la société. Au poème inerte, désincarné, enfermé dans l'espace du livre est alors opposé le poème " projeté » :

... pour un poème donc, debout dressé les pieds sur la page,

une page : tremplin pour quête d'oxygène,

projeté aussi, mobile, errant, à l'affût

à l'assaut des corps ${ }^{6} .$.

9 Heidsieck se met alors à produire des " poèmes-partitions ", confiant à l'écrit le seul rôle de notation d'un texte écrit pour sa lecture à voix haute. Muni, à partir de 1959, d'un magnétophone, il se met à les enregistrer, puis à capter des bruits extérieurs et faire du poème sur la bande le fruit d'un travail de montage. Mais la projection se fait aussi vers la salle, durant le temps de la lecture, qui lui fait substituer en 1963 l'expression «poésie action » à celle de " poésie sonore ». "Des lieux d'actions et d'auditions se substituent ainsi à la page écrite: scène, rue, salle d'écoute, studio, espace, quel qu'il soit. » Ses nouveaux véhicules sont « la voix, le cri, le geste, l'action, le bruit, le son, le silence, la bande magnétique, tout et n'importe quoi [...]» (Heidsieck 2001a: 72). "Dégutembergrisée », la poésie s'accorde aux évolutions de la société et des modes de communication, et se donne les moyens de l'invention, par la performance, d'un espace de «re-communication ». À la même époque, la poésie visuelle va dans le même sens, qui vise l'utilisation de supports comme l'affiche pour renouer avec une communication directe. Dès lors, ces poésies investiront d'autres circuits, allant des galeries d'art ${ }^{7}$ et musées aux festivals de musique électro-acoustique ${ }^{8}$.

Pour Chaton, il ne s'agit plus tant d'«arracher » la poésie du livre, que d'explorer les espaces ouverts par le geste effectué des décennies plus tôt. À l'instar de celle de son aîné, sa poésie se publie sur disque, et sur scène, et connaît des implémentations dans des secteurs institutionnels variés, de la maison d'édition au label de musique électronique (il rejoint Raster Noton en 2011), de la galerie d'art au concert rock (il fait la première partie des concerts du groupe The Ex en 2003), au théâtre ou encore à la fondation d'art contemporain (il crée, en 2015, RADIO pour la Fondation Vuitton9).

11 Son rapport au livre n'est pas polémique : les siens contiennent des textes imprimés qui ne sont pas les partitions des poèmes audibles sur le $\mathrm{CD}$ joint. La version sonore des pièces d'Événements 99 diffère partiellement du texte inscrit : le retour d'énoncés samplés, repris de gros titres de journaux («Washington déraille en Irak», entend-on dans "Événement $\left.\mathrm{n}^{\mathrm{o}} 1 »\right)$, vient rythmer, parasiter et interrompre à intervalles réguliers la lecture continue des textes "pauvres» (tickets de caisse, billets de train, carte d'identité, adresses sur enveloppes, etc.) collectés à la même date, seuls inscrits sur la page, produisant un effet de télescopage entre deux types d' "événements ». Vies des hommes illustres d'après les écrits d'hommes illustres ${ }^{10}$ va plus loin dans la divergence, se présentant comme un objet plurimédial : le livre s'accompagne d'un CD contenant les versions sonores de cinq pièces sur douze, dont certaines connaissent par ailleurs une implémentation scénique, lors de lectures publiques ( Vie de Christophe Colomb d'après Jules Verne $\left.{ }^{11} »\right)$, ou bien au théâtre (dont "Vie de Tibère d'après Suétone " écrite pour la pièce de Benoît Bradel, Napoli Express ${ }^{12}$ ). Les autres poèmes ne semblent avoir d'existence possible qu'imprimée : «Vie de Margaret Tatcher d'après Hugo Young " présente un graphique sur une longue bande de papier à déplier, et «Vie de René Descartes d'après Adrien Baillet », alterne 
d'imposantes formules mathématiques et leurs «tableaux d'équivalences », engageant le lecteur dans un jeu de reconstitution d'un texte pour le moins ardu. Mais l'imitation de l'inscription lapidaire, qui rend, par l'absence d'espacement entre les mots, la lecture de "Vie de Tibère d'après Suétone » problématique pour l'œil, connaît une version sonore, et une version scénique. Chaque manifestation matérielle du poème possède ainsi sa propre autonomie, où l'on s'éloigne de la stricte fonction partitionnelle qu'Heidsieck conférait à ses tapuscrits. La poésie de Chaton s'inscrit, aussi, hors du livre, sur des affiches, dans la série des Portraits ( 35 sérigraphies en format $176 \times 120 \mathrm{~cm}$ ), sur des magazines, prospectus, dépliants (série How is your life?), voire des mosaïques (pour Géographies, agrandissements de factures éditées dans différents pays), ou encore des vidéos ${ }^{13}$.

Moins que d'une rupture avec le livre, le mouvement impulsé conduit à le relativiser en le prenant pour ce qu'il est : un objet matériel. Il n'est donc pas paradoxal de constater que les «sonores» ou, les «poètes de la performance » continuent de produire des livres. Blaine n'a jamais cessé d'en publier, alors même qu'il proclamait l'« échec du livre » dans une performance-manifeste de 1971. Ce qu'il fustigeait, tout comme Heidsieck, était la fétichisation de l'objet, et la symbolique qui lui était associée.

\section{Résonances du ready-made ${ }^{14}$}

D'autres problèmes d'identification sont liés au contenu de ces poésies, soit qu'il n'y ait pas de texte lisible, lorsque la poésie est considérée comme art du verbe : Roubaud (2010) décrit « des "poètes" dont l'activité présentée au public comme poésie consiste à rouler en bas d'un escalier, à déchirer un gros annuaire téléphonique sur scène, à produire, électroniquement aidés, des séquences sonores inouïes et admirables, n'incluant pas un seul mot »; soit que le texte y apparaisse de piètre qualité : «Quand la langue est mise à contribution, dans un très grand nombre de cas le dépôt sur la page produit un texte médiocre", déplore-t-il. Là encore, une mise en perspective laisse apparaître la convergence des reproches : du ready-made au «non-art », la médiocre qualité formelle des œuvres est fustigée à renfort de descriptions jouant du procédé du regard naïf pour prouver que les œuvres ne «tiennent » pas sans l'appui d'un appareillage théorique. Ainsi J.-P. Domecq (2005: 7) énumère-t-il les « images de l'hallucination collective » au début de son pamphlet : « Un réfrigérateur trônant sur un coffre-fort - salle précédente : l'art c'est la vie en caractères d'enfant sur tableau noir - salle suivante : une toile fendue au rasoir [...]. »

14 Selon Genette (2004 : 88), un diagnostic de littérarité peut être soit thématique (c'est le critère de fictionnalité), soit rhématique, déterminant alors deux «modes de littérarité par diction » :

L'un (la poésie) est de régime constitutif: de quelque manière qu'on définisse la forme poétique, un poème est toujours une forme littéraire, parce que les traits formels (variables) qui le marquent comme poème sont, de manière non moins évidente, d'ordre esthétique. L'autre mode de diction (la prose non fictionnelle) ne peut être perçu comme littéraire que de manière conditionnelle, c'est-à-dire en vertu d'une attitude individuelle, comme celle de Stendhal devant le style du Code Civil. verbal. Envisagées selon ces critères, bien des occurrences de la poésie expérimentale 
depuis les années 1960 sortent de la littérature. Ce serait le cas pour une partie de l'œuvre de Jean-François Bory ${ }^{15}$ ou de Julien Blaine ${ }^{16}$, dont certains poèmes se composent exclusivement d'images. Dans la poésie sonore, les souffles, respirs, battements de cœur, bruits d'estomac, d'un Chopin ou les éructations de Dufrêne, nous en éloignent aussi. Chez Heidsieck, comme chez Chaton, on retrouve du non verbal. Sous l'influence de la musique concrète, Heidsieck intègre des bruits, enregistrés à même le réel, à ses poèmes : éclats de rires et cris d'enfants ( $\mathrm{D} 3 \mathrm{Z} »)$, échos de la circulation automobile ou du métro, etc. ("Coléoptères \& Co ", Le Carrefour de la Chaussée d'Antin). Chaton est plus économe dans son usage des bruits (mer démontée dans "Vie de Christophe Colomb ", cliquetis de machine à écrire dans "Vie de Freud») mais ses poèmes sont gorgés de chiffres, abréviations, symboles («Vie de Christophe Colomb» est un ensemble de coordonnées géographiques, en notation DMS - «Degré, Minutes, Secondes»). Dans un cas comme dans l'autre, ces éléments viennent s'associer à un matériau langagier qu'une approche strictement « littéraire », attachée au texte, viendrait seul sonder (voir Bobillot 1996).

Or dans bien des cas, celui-ci est composé d'autres textes, relevant de genres discursifs « routiniers", aux usages "balisés dans l'espace social» (Maingueneau 2004: 181), et distincts des genres auctoriaux dont la littérature fait partie. Ils relèvent, pour beaucoup d'entre eux, du ready-made ${ }^{17}$. Heidsieck utilise ainsi un exposé sur des techniques bancaires dans «B2-B3 », les titres du journal radiodiffusé dans «La Mer est grosse », ou encore le contenu de ses papiers d'identité dans "Qui je suis en une minute». Le déplacement ne vise cependant pas à conférer une "littérarité acquise " à l'élément prélevé, en exemplifiant ses propriétés esthétiques, ce en quoi il s'éloigne des entreprises modernistes ou surréalistes. Mais son indifférence esthétique ne rabat pas l'attention sur le seul geste dont il procède : il ne s'agit pas de réitérer les transgressions défiguratives de Dada. Il vise l'observation de quelques fragments du «corps social» qualifiés de «biopsies» par l'auteur, et relève à ce titre d'un usage documentaire. Si «dans notre espace littéraire paradigmatique, la littérarisation (l'extension du domaine littéraire) n'est jamais séparable d'une valorisation » (Hanna 2010 : 9), alors leur « littérarité » n'est pas même conditionnelle.

17 Le prélèvement est une constante de l'écriture de Chaton. Autoportraits (2003) s'ouvre sur "Autoportrait en complet noir », et se clôt sur "Autoportrait à l'écharpe rouge ». La convocation du modèle pictural prévue par le titre rhématique est confirmée par la série des compléments de noms qui indexent les accessoires dont s'accompagne le poète. Mais au lieu de l'image attendue se trouve un écrit: le premier est le relevé exhaustif des inscriptions présentes sur le passeport du poète ${ }^{18}$, façon de décliner son identité au seuil de l'entreprise, et le dernier propose la transcription d'un bilan sanguin, plongée littérale dans l'intérieur du poète, comme un clin d'œil au fait que, au cours de cette traversée, pas grand-chose de son intériorité ne nous aura été révélé. Au lieu des confidences attendues d'une entreprise potentiellement lyrique, les autres textes continuent en effet l'inventaire des écrits que le poète porte sur lui ou lit dans son entourage immédiat: tickets de caisse, paquets de cigarette, ticket de métro, cendrier, ou encore couvertures de livres dans " Autoportrait accoudé à la bibliothèque ». Se construit ainsi, peu à peu, un autoportrait métonymique, comparable aux « Portraits-Poubelles » d'Arman. Partant du constat que nos activités laissent un nombre considérable de traces écrites, le poète étend par la suite le procédé à d'autres, dans la série Portraits, d'où émergent des figures définies socialement comme «L'avocat », «L'ouvrier », etc. : 
Tous ces documents sont des écritures que nous partageons tous et dont on fait une lecture flash très particulière. On ne les lit pas vraiment, on les parcourt. Ce sont des textes qui nous sont très intimes, qui sont constituants de notre existence sociale. Mon travail consiste simplement à les cadrer pour constituer un récit, le récit d'une vie ou de l'inverse d'une vie, de ce qu'elle est ou de ce qu'elle n'est pas. (Chaton 2011) conceptuel, et reprenant les propositions de Kenneth Goldsmith à propos de l'« uncreative writing ", fondée sur le prélèvement, l'on pourrait alors parler à son propos d'« écriture conceptuelle », en ce que la dimension esthétique y passe au second plan, au profit de la mise en œuvre de procédures visant à interroger les conditions d'apparition de l'œuvre, et de la création de "pièces " (Binkley [1972] 1992) dont la dimension formelle n'a que peu de pertinence. Dès lors, «l'exemple de l'art plastique conceptuel suggère qu'en littérature également, la littérarité pourrait se situer ailleurs que dans l'objet lui-même (c'est-à-dire dans sa forme et son contenu)», mais par "la reconnaissance d'une intention littéraire qui pourrait être réalisée contextuellement » (Shusterman $2002: 68$ ). Un régime de littérarité «conceptuel », situé hors de la dichotomie fiction / diction, serait alors à penser. Cependant, l'expression trouve rapidement sa limite, autant que dans les arts plastiques où l'on a, à tort, assimilé ces productions à des œuvres de pur langage, mettant de côté ce qui pourtant reste une donnée fondamentale: leur matérialité.

\section{Poètes sans écriture?}

Corrélativement à leur absence supposée de valeur esthétique, la "médiocrité » des textes incriminés est rapportée à une absence d'exigence d'écriture. Usant de matériaux et moyens non répertoriés comme littéraires, ces poètes seraient sans écriture, comme ces «artistes sans art " (Rosenberg 1972: 12) dont la prolifération caractériserait l'art contemporain.

L'usage de matériaux préexistants tend à substituer à l'écriture telle que traditionnellement conçue une série de gestes semblant relever d'autre chose que d'elle, comme le suggère la recette de Tzara, "Pour faire un poème dadaïste ", suite de gestes enchaînés dans un protocole : d'ailleurs il ne s'agit pas d'écrire un poème mais de le faire. La substitution des ciseaux à l'instrument traditionnel d'écriture tient ainsi de 
l'éloignement de ce que Duchamp nomme "la main». La pluralisation des gestes et protocoles d'écriture est une caractéristique des avant-gardes historiques, et l'entreprise de démystification à laquelle Cadiot et Alféri (1995: 3) se livrent dans la RLG, résonne avec la première, lorsqu'ils entendent mettre à distance la mythologie de l'écriture lyrique, comme écriture frénétique sous l'emprise de l'inspiration: "On pourrait raconter l'écriture comme la construction d'un barrage, ou d'un moulin, ou d'un moteur. » L'index qui clôt le numéro décline à l'infinitif un ensemble d'opérations (" agglutiner, agrandir, anamorphoser, automatiser, baptiser, boucler, bricoler, cadrer, chiffrer, cliquer, compresser, couper... »). À un imaginaire romantique de l'écriture du jaillissement, ou à celui du labeur de l'écrivain penché sur sa table de travail, se substituent d'autres images. Vies..., tout en s'inscrivant dans une tradition littéraire et historique initiée par Plutarque, se compose ainsi de la redisposition de fragments puisés dans différents récits de vie. L'écriture ne consiste pas à effectuer un montage de type "cut-up ", mais à créer, pour chaque "vie", un dispositif unique : les citations de Margaret Tatcher sont par exemple insérées dans un tableau du cours de la bourse de Londres entre 1979 et 1988, faisant des paroles de la Première ministre l'unique facteur déterminant de ses variations journalières, rappel de l'ultra-libéralisme triomphant dont elle est le symbole.

L'importation dans l'espace d'écriture de dispositifs appartenant à des sphères non littéraires soulève également la question de la relation de ces poésies aux autres arts. Le partage des moyens ne relève pas d'une volonté d'aller vers ces arts, mais avant tout d'« élargir le langage poétique » (Castellin 1993 : IX). «Je ne suis pas du tout musicien »: ces affirmations répétées de la part de Heidsieck (1998: 25) comme de Chaton ne convoquent pas tant la " poésie » comme « effet fantôme » (Roubaud 2010) ou étiquetage institutionnel commode pour se maintenir artificiellement dans un champ, qu'elles ne mettent l'accent sur une différence dans la manière d'aborder des moyens communs. Et c'est bien, dans ce sens, d'écriture qu'il s'agit comme le souligne Chaton (2014:1181) :

Ce que Bernard Heidsieck ne cesse de nous dire, et de me dire à moi qui me dis poète sonore, c'est qu'il en va d'un autre régime de la poésie et de son dire dès lors qu'à ses outils d'écriture "gutembergisant» elle adjoint, [...] ceux d'autres écritures, radiophoniques, musicales ou électroniques.

Castellin (2001: 139) parle, à propos des poésies visuelles, de "désécriture ", ou d' écritures, insistant sur le fait que ces pratiques situent leur écart « en référence (critique) à l'histoire de la poésie ", donc à une certaine vision de l'écriture, et Spatola (1993: 67) d' "écritures technologiques ". L'utilisation de moyens techniques relève alors d'une attitude expérimentale au sens où il s'agit de les utiliser depuis la poésie. Chaton (2008: 88) précise ainsi ne pas avoir " de pratiques ou de manières de travailler de musicien ", ni de " connaissance aiguë de la fabrication du son », et utiliser « [s]on incompétence » : « Je vais à l'appropriation de l'outil de façon très empirique. » L'attitude de ces poètes est alors de l'ordre de l'expérimentation, "stratégie par laquelle l'incompétence ouvre la possibilité d'un nouveau régime de compétence» (During, Jeanpierre, Kihm et Zabunyan 2009 : 15).

Je ne fais que cadrer : je déplace un objet de son endroit d'inscription d'origine pour l'amener dans un autre endroit d'inscription. C'est mon seul geste ; ensuite, ce sont les éditeurs qui font que ça devient poésie, c'est le libraire qui accepte de diffuser l'éditeur qui fait que ça devient de la poésie, c'est le programme d'une manifestation quelconque qui, m'invitant, écrit que je fais de la poésie, etc. (Chaton 2013) 
'est cependant pas tout à fait certain que, à l'instar du ready-made que ces propos convoquent implicitement, l'entrée dans la chaîne institutionnelle provoque à elle seule le « devenir poésie » des matériaux prélevés. S'y intercale en effet, le poète y insiste par ailleurs, un travail d'écriture. Simplement, celle-ci ne porte pas directement sur la matière verbale, mais sur ses médiations, institutionnelles et matérielles.

\section{Écritures sonores}

Sortir du livre implique une évolution dans les modes de diffusion de la poésie, mais surtout leur conjugaison avec un «mode de faire» (Heidsieck 2001a: 85, 12), qui se traduit par le recours « à la plus large gamme [...] des possibilités techniques offertes par le magnétophone ». Chez Heidsieck, l'écriture du texte en est affectée. Outre des variations sur la voix, de multiples procédures de montage sont à l'œuvre : la suppression des souffles entre les mots ou groupes syntaxiques contribue à des accélérations rythmiques. La concaténation de matériaux hétérogènes, brefs échantillons de bruits divers (voiture qui freine, cri d'enfant, voix) sur un temps très court dans « La Convention collective» (1965) crée un effet de "photographie mentale». Enfin, l'écriture sonore s'enrichit des possibilités offertes par le mixage, en jouant, comme dans « B2-B3 » (1962), $\mathrm{du}$ déroulement simultané des pistes. L'absence d'affectation de l'écriture est, précisément, ce que le poète sonore (Heidsieck 2001a : 82) reproche aux lectures diffusées à la radio ou sur disque à l'époque :

Dieu sait pourtant - et les disques encore mieux, ou tant et tant, tant et tant de tentatives diverses, aussi, [...] ce cumul d'efforts déployés pour extraire du livre le poème écrit. Efforts vains. Ridicules et vains. La poésie écrite est faite pour rester couchée. C'est son destin. Qu'elle s'y tienne.

L'esthétique « lo-fi » de Chaton est avare en manipulations, mais le montage, le sample, les boucles, sont aussi à l'œuvre dans ses pièces. «Vie de Jésus-Christ d'après Ernest Renan » par exemple, se compose d'une longue liste d'actions attribuées au personnage, obéissant à la même structure syntaxique de base: "J.-C. a marché dans les rues de Nazareth. J.-C. a parcouru la Galilée... ». Celle-ci est cependant éclatée dans la version sonore, où les seuls verbes sont dits par le poète à un rythme très rapide, pendant qu'une multitude d'autres voix prennent en charge d'autres énoncés, créant un effet de polyphonie autour d'une figure centrale. Pour Chaton cependant, l'écriture se situe en deçà des manipulations, et commence dès qu'un système d'amplification est utilisé. À l'écoute des poèmes composant Événements 99, l'auditeur perçoit ainsi, d'abord, une voix à la diction rapide, monocorde et atonale, lisant les écrits prélevés. La dépersonnalisation de la voix met celle-ci en accord avec la pauvreté des matériaux, et la vitesse de diction finit par parasiter le contenu sémantique du texte, cédant la place à une sorte de litanie. L'écoute se fait alors par ponctions :

[...] ça crée une certaine lassitude auditive, du coup l'écoute se transforme et on arrive à cette idée de chant puis à partir de là on recrée une écoute visuelle : il y a des mots qui ressurgissent, on sort du texte, on y revient ; là, on peut commencer à se raconter des choses... (Chaton 2006)

Dans les pièces sonores de Vies..., les médiations techniques de la voix créent des effets de sens distincts. «Vie de Napoléon I d'après Stendhal» laisse entendre une voix radiophonique, qui semble énoncer les titres d'un journal perceptible seulement par bribes, au milieu d'un zapping radio provoqué par un changement rapide des fréquences. Le poème se donne comme partie du flot médiatique, indexant à la fois la célébrité 
légendaire d'un personnage et l'écriture journalistique de Stendhal. Cette voix nous parvient, dans "Vie de Christophe Colomb", par le biais d'une radio marine, en adéquation avec la nature du contenu textuel. Voix et diction, amplifiées, sont ainsi traitées comme médium à part entière, et les écrits collectés remédiés par ce biais libèrent des significations nouvelles.

\section{Écritures visuelles}

L'ensemble des médiations du texte peut ainsi faire l'objet d'une écriture, ce que certaines poésies visuelles ont appliqué au domaine de l'imprimé. Une grande partie de l'œuvre de Blaine traite de cette question, interrogeant, à travers le paradigme de l'empreinte comme écriture originelle, ce que peut être une forme pour l'offset. Il s'agit là aussi d'aborder un moyen technique comme moyen d'écriture, raison pour laquelle les poètes visuels ne travaillent, historiquement, pas ou peu avec des typographes. Dans l'œuvre de Chaton, l'importance de cette part tient d'abord au fait qu'il perçoit la «littérature pauvre » des imprimés quotidiens, comme "essentiellement visuelle», de l'ordre du "graphe ». Dans Événements 99 comme dans Autoportraits, la disposition graphique du texte, en ruban, implique une première appréhension visuelle, qui enclenche un mode de lecture par sauts, l'œil étant attiré, dans ce rectangle imprimé, par certains segments inscrits en capitales et/ou caractères gras. Les Autoportraits accentuent cette dimension, faisant signe vers le pictural via les titres, et le choix pour la série Portraits d'imprimer les textes en des sérigraphies de grand format, appelant la vision frontale du tableau ou de l'affiche entérine le paradigme pictural. Exposés en galerie, ces portraits convoquent l'image de l'écrit d'une manière qui prend l'art de l'affiche publicitaire pour contremodèle, en donnant à voir des blocs compacts, rappelant le rectangle typographique de la page imprimée, exhibant aussi, de ce fait, la transmédiation à l'œuvre. Les écrits prélevés font en effet, dans le transfert, l'objet d'une neutralisation typographique visant à unifier, visuellement, un ensemble textuel hétérogène : à créer une cohérence qui permette de faire portrait.

La spatialisation du texte et les effets de transmédiation sont aussi au cœur de Vies..., qui prend pour matériaux d'autres écrits dont les titres sont indiqués en fin d'ouvrage. Le terme est à prendre ici dans un sens pleinement matériel. Ainsi «Vie de Le Caravage d'après Arnaud Labelle-Rojoux ", reproduit des pages de l'édition Al Dante de Récits de la vie de Michelangelo Merisi dit Le Caravage, à l'aide d'une photocopie qui, par sa médiocrité, laisse ses traces, et, par là, se montre. Le cadrage, purement graphique, ne tient aucun compte de la syntaxe ni du sens, à l'instar d'un « Dépôt » rochien. Le récit de vie, devenu partiellement illisible, prend la place de l'œuvre picturale légendée pour en composer une nouvelle version, nourrie d'un des récits contribuant à en perpétuer l'existence. Dans les autres pièces, les textes sont redisposés ou réécrits selon des dispositifs empruntés à d'autres régimes médiatiques, qui correspondent à autant de supports, dont l'expression graphique garde mémoire: inscription lapidaire («Vie de Tibère »), tapuscrit de machine à écrire ("Vie de Sigmund Freud d'après Sigmund Freud »), SMS (la "Vie de Dante Alighieri d'après Giovanni Boccacio »), etc. Comme dans les Portraits, la vie se compose par les traces inscrites, et les différentes opérations de transmédiation à l'œuvre recréent des "vies» aussi en ce qu'elles en sont des réinscriptions. 


\section{Écritures de la performance}

31 L'écriture se fait, enfin, dans et par la performance. Dans « Newspaper ", réalisé avec Andy Moor ${ }^{19}$, les dimensions visuelle ${ }^{20}$ et gestuelle s'adjoignent à la dimension sonore pour engager des sens nouveaux. Sur scène aux côtés du guitariste, Anne-James Chaton commence par lire au micro les gros titres du journal du jour qu'il tient devant lui, pendant que la guitare, jouée en staccato, semble réagir à la voix. L'ensemble est décousu, rendant sensible l'écriture par prélèvement qui s'opère en direct. Puis, la guitare occupe seule l'espace sonore, pendant que le poète se rend derrière l'ordinateur installé sur le bureau, sur scène, afin de retraiter le matériau lu au micro et enregistré. La voix enregistrée se décroche alors de son locuteur physique, et s'autonomise, devenant le matériau de samples dont la superposition finit par créer un effet de polyphonie chaotique, auxquels des riffs répondent. Après avoir jeté au sol des bandes de papier, le poète en ramasse au hasard et lit au mégaphone les citations également extraites de journaux qu'elles contiennent. Ainsi amplifiée, la voix fait retour au premier plan mais le médium a un son granuleux qui rend le contenu difficilement intelligible et attire l'attention sur la matière sonore. Une sorte de lutte s'instaure, l'ensemble créant un effet de saturation, et une impression de chaos, d'une avalanche d'informations rendues inintelligibles par leur abondance même. Au sein de ce dispositif complexe, les objets, gestes multiples d'écriture et instruments de la phono-techné se conjuguent pour exhiber une écriture seconde, qui s'effectue dans le temps de la performance.

Le mode d'écriture résultant, ici, de la collaboration avec un musicien d'improvisation, accentue quelque chose qui se joue de manière constante dans l'œuvre de Chaton, y compris dans ses dispositifs les plus simples, et constitue la lecture publique en écriture. Opérant par ponctions, le poète ne lit pas exactement le texte imprimé, reconfigurant de la sorte les matériaux, mais l'écriture se situe également dans les médiations de la phonotechné, et l'ensemble des « techniques du corps » (Mauss 1934) déployées sur la scène. Il est à cet égard parlant de noter que le poète récuse le terme de "performance " pour parler de «lecture ». S'il évoque pour cela le caractère improvisé de la première pour l'opposer à la préparation minutieuse de la seconde, ce rejet serait aussi à chercher dans une volonté de maintien dans le paradigme littéraire, en utilisant le couple "écriture / lecture ", mais aussi dans l'importance conférée à la spécificité de chaque médium. "Je suis d'une certaine façon matérialiste, jusque dans mon écriture, et je crois beaucoup à cette antécédence de l'écrit sur le dire " (Chaton 2008). Partant, la présence constante d'un support imprimé sur la scène contribue à la mise en évidence d'une confrontation des écritures :

Loin de prétendre à une quelconque immédiateté, l'action du poète met en branle la mécanique d'écriture; elle mixe les supports sur scène, sans jamais se départir de son texte, se rendant ainsi un peu plus étrangère au sentiment de soi de l'oralité. La feuille ou le livre, tenu à la main ou posé sur la table, sur un pupitre, ou le long rouleau de Vaduz, rappelle, à qui veut bien s'en souvenir, d'où vient la poésie sonore. (Chaton $2014: 1181$ )

Ce rapport à l'écrit rejoint celui de Heidsieck chez qui la partition tapuscrite fait partie intégrante du dispositif prévu par la performance (Théval 2018), comme le montre de façon spectaculaire $V a d u z$, durant lequel le poète déroule, à mesure de la lecture, un long "papyrus» sur lequel s'inscrit l'interminable liste de noms d'ethnies présentes «tout autour de Vaduz ». L'imprimé peut d'ailleurs s'y décrocher de son statut de partition, et 
pour «H1-H2. Le Quatrième Plan", le poète ne lit pas le texte diffusé par les hautparleurs, se contentant de distribuer au public « des masses de statistiques inscrites sur feuilles d'ordinateurs ou rouleaux de machines à calculer » (Heidsieck 2001b : 60).

Le régime allographique (Goodman 1990) de la littérature, présupposé par l'hypothèse d'un « dépôt » possible du texte sur la page, vacille, qui suggère qu'un texte est identique à lui-même quels que soient sa graphie et son support d'inscription, idée elle-même adossée à une conception phonocentriste du langage niant la nature graphique du signe (Baetens 1988). Les différents modes d'expression médiatique de ces poésies produisent ainsi un déplacement, autorisant l'extension de la notion d'" écriture ", qui redéploie dans ses utilisations son sens matériel pour le lier, pleinement, à son sens d'acte créateur.

\section{Conclusion}

Sortie du livre, incursion du «n'importe quoi », usage expérimental des technologies, les pratiques ici évoquées semblent ainsi à bien des égards sortir de la littérature envisagée selon l'actuel paradigme. Faut-il, pour autant, les en exclure, et en déduire l'existence d'une poésie "non littéraire ", prenant acte de sa désinstitutionnalisation pour en faire par exemple une branche de l'art contemporain? Mais n'est-ce pas en manquer une partie des enjeux propres, qui se dessinent précisément au regard du champ de la littérature? Et comment désigner ces objets? La métaphore ufologique trouve vite ses limites, et la notion foucaldienne de «dispositif» pour opérante qu'elle soit dans la description du fonctionnement de ces objets, comporte le risque de désancrer ces pratiques d'une histoire littéraire dont elles font, pourtant, partie intégrante ${ }^{21}$. Certes, ces dispositifs sont « indescriptible[s] dans le cadre d'une théorie littéraire axée sur le double critère fiction / diction" (Hanna 2010: 15), engoncée dans un logocentrisme qui ne lui permet pas de prendre en considération ces éléments non linguistiques. La spatialisation des textes poétiques n'est cependant plus tout à fait « un point aveugle de la critique littéraire ", et plusieurs travaux ont été entrepris depuis le début des années $1980^{22}$ en ce sens. L'extension manifeste de ces pratiques semble devoir amener à une extension du domaine de la littérature, impliquant une modification du paradigme en cours, que les récents développements des études en intermédialité peuvent favoriser.

Celle-ci se définit comme une approche étudiant la manière dont les «matérialités de la communication font partie du travail de signification et de référence »: "Les effets de sens sont aussi des dispositifs sensibles " (Méchoulan 2003 : 10). Relevant d'une approche synchronique, elle s'attache aux phénomènes de coprésence de plusieurs médias. On rejoint alors les théories de l'« intermedia» formulées par Dick Higgins (1983) pour décrire les productions Fluxus, mais aussi celles de la poésie expérimentale. Elle peut, également, relever d'une approche diachronique, évaluant alors les phénomènes de transfert d'un média à l'autre, pour tenter de "saisir la manière dont une forme singulière est liée à d'autres formes qui lui sont contemporaines ou antérieures » (Besson 2014), et rendre compte des effets liés à la transmédiation. Partant, elle permet d'appréhender «la possibilité qu'une œuvre [...] prenne corps dans une variabilité de dispositifs formels, techniques, médiatiques, matériels et qu'en conséquence sa perception soit mobile et ses significations naturellement d'autant plus ouvertes » (Robic 2011 : 9). Cette migration, constitutive de la poésie expérimentale, est partie intégrante de nombreuses œuvres contemporaines qui intègrent ces paramètres dans leur fonctionnement esthétique. À charge aux études littéraires de ne pas les ignorer. 


\section{BIBLIOGRAPHIE}

Baetens, Jan, 1988, « Autographe / allographe (à propos d'une distinction de Nelson Goodman) », Revue philosophique de Louvain, vol. 86, nº 70, p. 192-199.

Besson, Rémy, 2014, « Prolégomènes pour une définition de l'intermédialité à l'époque contemporaine ", [En ligne, Rapport], https://hal-univ-tlse2.archives-ouvertes.fr/hal-01012325, consulté le 14 juin 2018.

Binkley, Timothy, [1972] 1992, « Pièces : contre l'esthétique », dans G. Genette (dir.), Esthétique et poétique, Paris, Seuil, p. 33-66.

Bobillot, Jean-Pierre, 1996, Bernard Heidsieck : poésie action, Paris, Jean-Michel Place.

Castellin, Philippe, 1993, « Préface », Adriano Spatola, Vers la Poésie totale, Marseille, Via Valeriano.

Castellin, Philippe, 2001, DOC(K)S : mode d'emploi, Romainville, Al Dante.

Castellin, Philippe, 2009, « "La poésie est sans épithète”, un entretien avec Philippe Castellin par Alexandre Gherban », 16 février, Poezibao, [En ligne], http://poezibao.typepad.com/ poezibao/2009/02/la-poésie-est-sans-épithète-un-entretien-avec-philippe-castellin-paralexandre-gherban.html, consulté le 14 juin 2018.

Cauquelin, Anne, 1996, Petit traité d'art contemporain, Paris, Seuil, coll. « La couleur des idées ».

Cadiot, Olivier et Alféri, Pierre, 1995, Revue de littérature générale, $\mathrm{n}^{\circ} 1$, « La mécanique lyrique ».

Cadiot, Olivier et Alféri, Pierre, 1996, Revue de littérature générale, n 2, « Digest ».

Chaton, Anne-James, 2006, « Entretien avec Anne-James Chaton », Le Sofa, n 1, [En ligne], http:// www.lesofa.org/entretienajchaton1.html, consulté le 14 juin 2018.

Chaton, Anne-James, 2008, « Entretien avec Jules Julien », Musique Action - Défrichage sonore, entretiens autour du festival, Marseille, Le Mot et le reste.

Chaton, Anne-James, 2011, « Rencontre avec Anne-James Chaton », Hartzine, [En ligne], http:// www.hartzine.com/rencontre-avec-anne-james-chaton/, consulté le 14 juin 2018.

Chaton, Anne-James, 2013, « Archéologie du ticket de caisse », Vacarme, n 65, [En ligne], http:// www.vacarme.org/article2282.html, consulté le 14 juin 2018.

Chaton, Anne-James, 2014, «L'écriture de la voix », Bernard Heidsieck : les tapuscrits, Dijon, Nice, Les Presses du réel, Villa Arson, p. 1180-1182.

Cohen, Nadja, Pardo, Céline et Reverseau, Anne, 2012, Poésie et médias, Paris, Nouveau Monde. Domecq, Jean-Philippe, 2005, Artistes sans art ?, Paris, 10/18.

During, Elie, Jeanpierre, Laurent, Kihm, Christophe, Zabunyan, Dork (dir.), 2009, In Actu. De l'expérimental dans l'art, Dijon, Les Presses du réel.

Garnier, Pierre, 1968, Spatialisme et poésie concrète, Paris, Gallimard.

Genette, Gérard, 2004, Fiction et diction, Paris, Seuil, coll. « Points / Essais ».

Gleize, Jean-Marie, 2009, Sorties, Paris, Questions théoriques, coll. « Forbidden beach ». 
Goodman, Nelson, 1990, Langages de l'art, Paris, Jacqueline Chambon.

Hanna, Christophe, 2010, Nos documents poétiques, Paris, Questions théoriques, coll. « Forbidden beach ».

Heidsieck, Bernard, 1998, Interview, DOC(K)SON, série 3, nº 17/18/19/20.

Heidsieck, Bernard, 2001a, Notes Convergentes, Romainville, Al Dante.

Heidsieck, Bernard, 2001b, Partition V, Bordeaux, Le Bleu ciel.

Heidsieck, Bernard, 2004, « Notes a posteriori », Derviche/Le Robert, Romainville, Al Dante.

Higgins, Dick, 1983, « Some Thoughts on Intermedia poetry », dans F. Janicot, Poésie en action, Issy-les-Moulineaux, Loques, NèPE, p. 63-72.

Lebovici, Franck, 2007, Des documents poétiques, Paris, Al Dante, Questions théoriques, coll. « Forbidden beach ».

Lecercle, Jean-Jacques et Shusterman, Ronald, 2002, L'Emprise des signes, débat sur l'expérience littéraire, Paris, Seuil.

Maingueneau, Dominique, 2004, Le Discours littéraire, Paris, Armand Colin, coll. « U ».

Mauss, Marcel, [1934] 1936, « Les techniques du corps ", Journal de psychologie, vol. 32, n 3-4.

[Édition électronique par J. M. Tremblay, 2002 : http://classiques.uqac.ca/classiques/ mauss_marcel/socio_et_anthropo/6_Techniques_corps/Techniques_corps.html, consulté le 14 juin 2018.

Méchoulan, Éric, 2003, «Intermédialités : le temps des illusions perdues », Intermédialités, $\mathrm{n}^{\circ} 1$, p. 9-27.

Robic, Jean-François (dir.), 2011, Intermédialité. Partage et migrations artistiques, Strasbourg, Université de Strasbourg, coll. « Cahiers recherche».

Rosenberg, Harold, 1972, La Dé-définition de l'art, Nîmes, Jacqueline Chambon.

Rosenthal, Olivia et Ruffel, Lionel, 2010, « Introduction », Littérature, n 160, « La littérature exposée. Les écritures contemporaines hors du livre », p. 3-13.

Roubaud, Jacques, 2010, « Obstination de la poésie », Le Monde Diplomatique, janvier, p. 23.

Ruffel, David, 2010, « Une littérature contextuelle », Littérature, nº 160, p. 61-73.

Spatola, Adriano, 1993, Vers la poésie totale, présenté, annoté et traduit par Philippe Castellin, Marseille, Via Valeriano.

Théval, Gaëlle, 2018, « Scénographies de l'écrit dans les performances poétiques de Bernard Heidsieck », dans O. Penot-Lacassagne et G. Théval (dir.), Poésie et performance, Nantes, Cécile Defaut, p. 117-132

Vallier, Dora, 1969, « Art, anti-art et non-art », La nouvelle revue française, $\mathrm{n}^{\circ} 201$.

\section{NOTES}

1. Loin d'être une notion stabilisée, le terme est utilisé ici pour désigner les pratiques aussi appelées « anti-art », dans la lignée de Dada, comme celles de Fluxus.

2. Les ruptures soulignées par exemple par Ruffel $(2010: 66)$ détaillant ce qui fait «sortir» les pratiques des années 1990 de la littérature s'émoussent lorsqu'on les confronte aux pratiques expérimentales, lorsqu'il s'agit, par exemple, de « remiser la lecture d'auteur qui ne soit pas en 
elle-même un acte artistique ainsi que les lectures déclamatoires des comédiens sacralisant le texte poétique ", raison d'être, pour Heidsieck, de la poésie sonore qui se construit précisément en opposition à l'une comme à l'autre, ou bien de proposer des poèmes photographiques, dessins poèmes, etc., caractéristiques de la poésie visuelle.

3. Entretien pour le documentaire Poésie action : Variations autour de Bernard Heidsieck, 2014, AnneLaure Chamboissier, Philippe Franck (réal.), a.p.r.e.s. éditions, cnap.

4. «Je me suis tourné vers la poésie sonore en partie du fait de ma bibliothèque personnelle, mais surtout parce que j'ai assisté en 1994 à une lecture de Bernard Heidsieck (il lisait son tube, Vaduz )$»($ Chaton 2013).

5. http://aj.chaton.free.fr.

6. Manifeste de 1961.

7. La première diffusion publique d'une œuvre sonore de Heidsieck («D3Z ») en 1961, a lieu dans la Galerie internationale d'art contemporain, à l'ouverture de l'exposition « Sept métasignes sur la fleur» de Jean Degottex. Citons aussi l'accueil par le Musée d'art moderne du Domaine Poétique, au sein de la section « Arts du langage » de la Biennale de Paris en 1963.

8. Le Festival international de musique expérimentale de Bourges ("Synthèse »), accueille par exemple en 1975 Chopin et Heidsieck : ce dernier y est lauréat du troisième concours de musique électroacoustique pour Vaduz.

9. http://www.fondationlouisvuitton.fr/radio.html.

10. 2011, Al Dante, désormais abrégé Vies...

11. Une lecture de 2011 est visible à cette adresse : https://vimeo.com/23976709 (consulté le 14 juin 2018).

12. Un diaporama est visible à cette adresse : https://vimeo.com/86694761 (consulté le 14 juin 2018).

13. Dont « Princess in a car », avec Andy Moor, publié sur YouTube : https://www.youtube.com/ watch?v=rjiRsAd-B4k\&feature=youtu.be (consulté le 14 juin 2018).

14. Nous empruntons l'expression au titre de l'ouvrage de Thierry de Duve, Résonances du readymade, Nîmes, Jacquelines Chambon, 1989, coll. « Rayon Art ».

15. Voir par exemple l'anthologie Post-scriptum, Paris, Losfeld, 1970.

16. Voir notamment 13427 Poëmes métaphysiques, Paris, Éditeurs évidants, 1986.

17. Sur ce point je me permets de renvoyer à mon ouvrage, Poésies ready-mades, $\mathrm{XX}^{e}-\mathrm{XXI}^{e}$ siècles, Paris, L'Harmattan, 2015, coll. « Arts et médias ».

18. La proximité avec "Qui je suis en une minute» d'Heidsieck, est évidente, soulignant la parenté des deux poétiques.

19. Enregistrée sur disque après son élaboration scénique, une version est parue en 2008 sur le label Unsounds, sous le titre « Le Journaliste ».

20. Description fondée sur une captation vidéo réalisée en 2009, lors du festival «Paris en toutes lettres », le 5 mai 2009, https://www.youtube.com/watch?v=hX6X0He7IU4 (consulté le 14 juin 2018).

21. Qui se réenvisage, précisément, à la lumière de ses rapports aux médias. Voir Cohen, Pardo et Reverseau (2012).

22. Voir les travaux de et autour de Anne-Marie Christin et le Centre d'étude de l'écriture et de l'image: http://www.ceei.univ-paris7.fr, ainsi que les propositions de J.-P. Bobillot sur la «médiopoétique ». 


\section{RÉSUMÉS}

À la lumière des œuvres de deux poètes de générations différentes, Bernard Heidsieck et AnneJames Chaton, cet article se propose d'évaluer la dette des pratiques contemporaines aux poésies expérimentales. Dans quelle mesure la «non-littérature» conduit-elle à une révision du paradigme littéraire, révision sans laquelle les pratiques actuelles sont difficilement appréhendables?

Studying the works of two poets from different generations, Bernard Heidsieck and Anne-James Chaton, this article intends to evaluate the debt of contemporary practices to experimental poetry. How did "non-literature" lead to a revision of the literary paradigm?

\section{INDEX}

Mots-clés : Chaton (Anne-James), Heidsieck (Bernard), poésie expérimentale, poésie sonore, poésie visuelle, écriture conceptuelle, performance, dispositif, ready-made, intermédialité, nonart, non-littérature

Keywords : experimental poetry, visual poetry, sound poetry, performance art, ready-made, intermediality, non-art, non-literature, uncreative writing, Chaton (Anne-James), Heidsieck (Bernard)

\section{AUTEUR}

\section{GAËLLE THÉVAL}

Université Sorbonne Nouvelle - Paris 3, Thalim (UMR 7172) 